\title{
Dispositivos Flexíveis de Monitoramento de pH e de Deflexão Mecânica à Base de Polianilina
}

\author{
Silmar A. Travain, Rodrigo F. Bianchi \\ Departamento de Física - UFOP
}

Enia M. L. Colella, Adnei M. de Andrade

Departamento de Engenharia de Sistemas Eletrônicos, EPUSP

\author{
José Alberto Giacometti \\ Faculdade de Ciências e Tecnologia, UNESP
}

\begin{abstract}
Resumo: Nesse trabalho descrevemos a arquitetura e a operação de filmes finos de polianilina (PAni) usados como elemento ativo de sensores flexíveis de $\mathrm{pH}$ e de deformação mecânica. Tais dispositivos apresentam grande interesse tecnológico devido aos seus baixos custos de produção e à possibilidade de integração com outros sistemas orgânicos e inorgânicos, incluindo transistores, diodos e baterias. Filmes finos de PAni foram preparados pelo método de polimerização in-situ sobre microeletrodos de $\mathrm{Cr} / \mathrm{Au}$ previamente depositados sobre substratos de polietilenoteraftalato (PET). As características elétricas do sistema foram investigadas como função do nível de dopagem da PAni. Os dispositivos apresentaram boa resposta elétrica para diferentes soluções ácidas e básicas, bem como para pequenas variações de deformações mecânicas.
\end{abstract}

Palavras-Chave: Condutividade elétrica, deflexão, dispositivo, pH, polianilina.

\section{Flexible Polyaniline Devices for Strain Gauge and pH Monitoring Applications}

Abstract: In this work we describe the design and operation of thin polyaniline (PAni) films as active material for strain gauge and $\mathrm{pH}$-monitoring flexible electronic devices. These devices are interesting due to their low cost and possible integration with other organic or inorganic systems, including diodes, transistors and batteries. Thin PAni films were prepared using the in-situ polymerization method on interdigitated chromium-gold microelectrodes previously deposited on poly(ethylene terephthalate) - PET substrates. The electrical characteristics of the polymer devices were obtained as a function of the PAni doping level. The devices showed high sensitivity for small strains and acid/base solutions.

Keywords: Display, deflection, polyaniline, electrical conductivity.

\section{Introdução}

Até meados dos anos 1970 os polímeros se destacavam na área de materiais pelas suas boas capacidades de isolamento elétrico e de versáteis propriedades mecânicas. Entretanto, após a descoberta da alta condutividade do poliacetileno dopado em $1977^{[1]}$, os polímeros começam a adquirir o status de materiais com propriedades eletrônicas. Torna-se possível alterar, reversivelmente, a condutividade elétrica desses materiais variando-a desde um valor muito baixo (isolante), até valores típicos dos metais. Nos anos que se seguiram até os dias de hoje, dezenas de outros polímeros foram sintetizados, apresentando comportamento isolante-condutor similar ao do poliacetileno ${ }^{[2]}$. Dentre esses polímeros, os que têm sido mais amplamente estudados são as polianilinas, os polipirróis, os politiofenos, os poli $(p \text {-fenilenos) e os poli( } p \text {-fenilenivinilenos })^{[2-6]}$.

Um exemplo da rápida evolução desta área foi o desenvolvimento, no começo da década de 1980 , de uma bateria usando eletrodos poliméricos, que serviu futuramente de base para o desenvolvimento de baterias recarregáveis ${ }^{[6]}$. Atualmente, diodos, transistores, sensores de gases, sensores químicos e biológicos, dosímetros, aplicações em eletrônica biomolecular, músculos artificiais, diodos emissores de luz, displays luminosos, células fotovoltaicas são outros exemplos de aplicações dos polímeros eletrônicos ${ }^{[5-11]}$. Nesse contexto, o estudo e a caracterização de filmes e sistemas orgânicos à base de polianilina (PAni) para aplicações em dispositivos eletrônicos têm despertado grande interesse tecnológico ${ }^{[12,13]}$. Isso ocorre pelo fato desse polímero apresentar, além da possibilidade de controle da sua condutividade elétrica por meio da exposição a soluções ácidas ou básicas, baixos custos de produção, solubilidade em diversos solventes orgânicos, facilidades de processamento e de manufatura na forma de filmes finos e, finalmente, estabilidades térmica, química e elétrica ${ }^{[14]}$. Em outras palavras, propriedades físico-químicas e características mecânicas e elétricas promissoras para o desenvolvimento de dispositivos eletrônicos inovadores.

Autor para correspondência: Rodrigo F. Bianchi, Instituto de Ciências Exatas e Biológicas, Departamento de Física, Universidade Federal de Ouro Preto, CEP: 35400-000, Ouro Preto, MG, Brazil.E-mail: bianchi@iceb.ufop.br 
Neste trabalho são apresentadas as etapas de preparação e a caracterização elétrica de dispositivos flexíveis à base de polianilina e de polietilenoteraftalato para aplicações em sensores de monitoramento de pH e de deflexão mecânica.

\section{Experimental}

Para realização deste trabalho foram preparados sistemas eletrônicos a partir da deposição de um filme fino de polianilina (PAni), via técnica de deposição in-situ, sobre microeletrodos de cromo e ouro previamente depositados sobre substratos de polietilenoteraftalato (PET). A técnica de deposição in-situ consiste em depositar, durante o processo de polimerização da anilina, filmes de PAni sobre diferentes substratos com controle de uniformidade e aderência ${ }^{[15-17]}$. Os microeletrodos de $\mathrm{Cr} / \mathrm{Au}$ sobre PET, por sua vez, foram preparados a partir de técnicas de litografia convencionais disponíveis na literatura ${ }^{[18,19]}$. A Figura 1 mostra a geometria e as especificações dos microeletrodos preparados. Para tanto, foram utilizados filmes de PET com espessura da ordem de $170 \mu \mathrm{m}$ e área de $2,54 \times 2,54 \mathrm{~cm}^{2}$, previamente limpos com

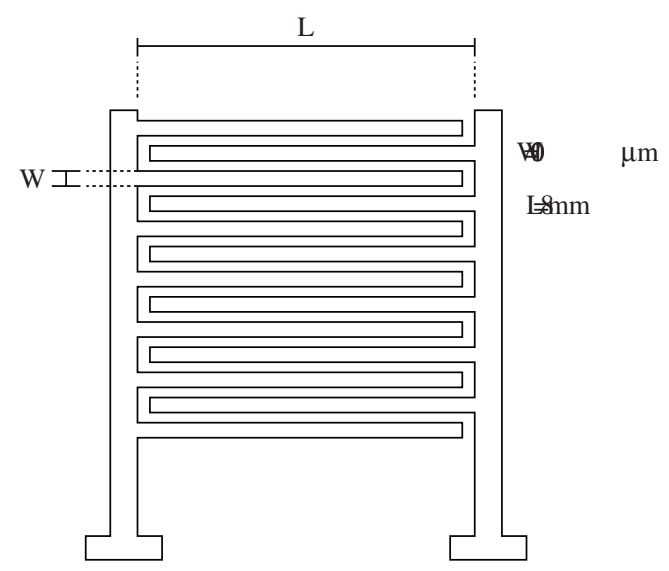

(a)

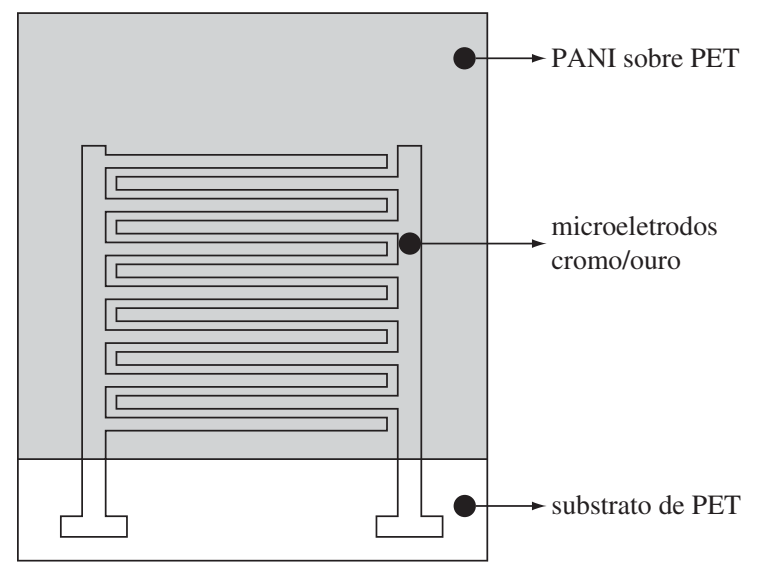

(b)

Figura 1. Estrutura dos sistemas preparados. a) eletrodos interdigitados sobre substrato de PET (comprimento $-\mathrm{L}=8 \mathrm{~mm}$, distância $-\mathrm{W}=100 \mu \mathrm{m}$ e altura - $100 \mathrm{~nm}$ ); e b) eletrodos interdigitados sobre substrato de PET recobertos pelo filme de PAni. água destilada e detergente Extran (diluído 3\%). Finalmente, a espessura dos filmes de PAni $(\sim 100 \mathrm{nn})$ foi obtida a partir de degraus existentes entre as superfícies desse polímero e do substrato de PET em imagens de microscopia de força atômica. As imagens foram obtidas com o equipamento $\mathrm{Na}-$ noscope III da Digital Instruments em modo contato.

\section{Caracterização dos Filmes e Dispositivos Poliméricos}

\section{Filmes de PAni}

Neste trabalho a resistividade elétrica dos filmes de polianilina foi investigada por meio de duas técnicas experimentais complementares: uma de análise típica de resistividade de superfície e outra de resistividade de volume. A primeira, de superfície, foi realizada a partir do método de quatro pontas ${ }^{[20]}$, aplicando-se, portanto, uma corrente constante e medindo-se a tensão entre dois pontos do filme. Nesse caso, foi utilizado o equipamento VEECO FPP-100. Já a segunda técnica, de volume, foi realizada com o equipamento HP4156A, no intervalo de tensão de 0 a 3 Volts. A técnica resume-se em aplicar a tensão e medir a corrente numa estrutura de placas planas e paralelas. No nosso caso, tal estrutura foi obtida a partir da utilização dos microeletrodos (Figura 1a) previamente depositados sobre o PET e preenchidos com PAni (Figura 1b). Os resultados obtidos pelas duas técnicas mostraram que a resistividade elétrica do filme de PAni $\left(\sim 5 \times 10^{-3} \Omega \mathrm{m}\right)$ é da ordem dos valores obtidos para vários semicondutores inorgânicos ${ }^{[21]}$, bem como da ordem dos valores da resistividade de volume obtidos de filmes auto-sustentáveis desse polímero ${ }^{[22,23]}$. Logo, pode-se concluir que os filmes finos preparados pela técnica in-situ não apresentam distinção entre as resistividades elétricas de volume e de superfície, ao contrário do que ocorre em filmes espessos, onde os diferentes estados de oxidação nas suas superfícies mudam drasticamente esses valores e o comportamento da resistividade elétrica no volume e na superfície dos $\operatorname{mesmos}^{[22,24]}$.

\section{Sensor de $\mathrm{pH}$}

O objetivo desta aplicação é a obtenção de um sistema de monitoramento de $\mathrm{pH}$ com princípio de operação baseado na mudança da resistividade elétrica da PAni quando exposta à diferentes soluções ácidas ou básicas. Para a confecção destes dispositivos utilizou-se a estrutura mostrada na Figura 1b. Essas estruturas foram imersas em soluções de $\mathrm{HCl}$ e $\mathrm{NH}_{4} \mathrm{OH}$ com diferentes valores de $\mathrm{pH}$, monitorados com um pHmetro Analion - PM608. Medidas de variação da corrente do dispositivo em função do tempo e do $\mathrm{pH}$ foram obtidas por meio de um circuito eletrônico simples e composto por uma fonte de tensão e por um amperímetro, mantendo-se o campo elétrico inferior a $10^{4} \mathrm{~V} / \mathrm{m}$. Finalmente, para a caracterização do dispositivo, foi adotado o seguinte procedimento experimental: 
- O dispositivo polimérico foi imerso na solução de pH = 1 (solução de referência), para padronizar as condições iniciais de operação do sensor;

- Uma vez imerso na solução de referência, o mesmo foi mantido em repouso por 1 min para que o sistema atingisse seu equilíbrio químico;

- Após atingir o equilíbrio, o dispositivo foi alimentado com $3 \mathrm{~V}$ e sua curva corrente $v s$. tempo foi registrada até que sua corrente atingisse seu valor de saturação; e

- O dispositivo foi então curto-circuitado, para eliminar possíveis efeitos de memória, e novamente imerso na solução de referência para futura análise de outras soluções ácidas ou básicas repetindo-se, para tanto, os 3 passos anteriores.

\section{Sensor de deflexão mecânica}

Para o sensor de deflexão mecânica foi proposta a utilização do mesmo dispositivo usado como sensor de $\mathrm{pH}$ que, em vez de ser exposto a diferentes soluções ácidas ou básicas, foi pressionado mecanicamente. Para atingir esse objetivo um sistema simples e eficiente, composto por uma morça e um transferidor, foi preparado tal como mostrado na Figura 2, onde os eletrodos são colocados na parte convexa do substrato de PET. O sistema permitiu a variação do ângulo de deflexão $(\theta)$ do dispositivo de 0 a $55^{\circ}$, enquanto um circuito eletrônico composto por uma fonte de $3 \mathrm{~V}$ e um amperímetro foi usado para monitorar a corrente do mesmo até que essa atingisse seu estado estacionário por volta de $5 \mathrm{~min}$.

\section{Resultados e Discussão}

A Figura 3 mostra a evolução do tempo de estabilização da corrente $\left(\mathrm{T}_{\text {est }}\right)$ que flui pelo dispositivo de PAni quando exposto a diferentes valores de $\mathrm{pH}$. Os resultados mostram que $\mathrm{T}_{\text {est }}$ é diretamente proporcional ao $\mathrm{pH}$ variando de $120 \mathrm{~s}$ para $\mathrm{pH}=2$ e atingindo $260 \mathrm{~s}$ para valores de $\mathrm{pH}$ maiores que 8. Logo, o modo de operação do sensor deve levar em consideração que o tempo de resposta do mesmo é de $260 \mathrm{~s}$, uma vez que, nesse intervalo, o sistema já atingiu seu estado de equilíbrio para todos os valores de $\mathrm{pH}$ investigados. Esse resultado é bastante útil para a confecção dos circuitos de controle do dispositivo, pois permite associar o tempo de resposta do sistema com os possíveis circuitos elétricos a serem

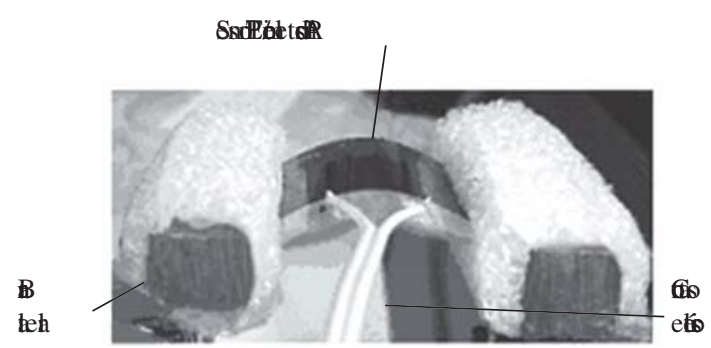

Figura 2. Aparato experimental utilizado na caracterização do sensor de deflexão mecânica.

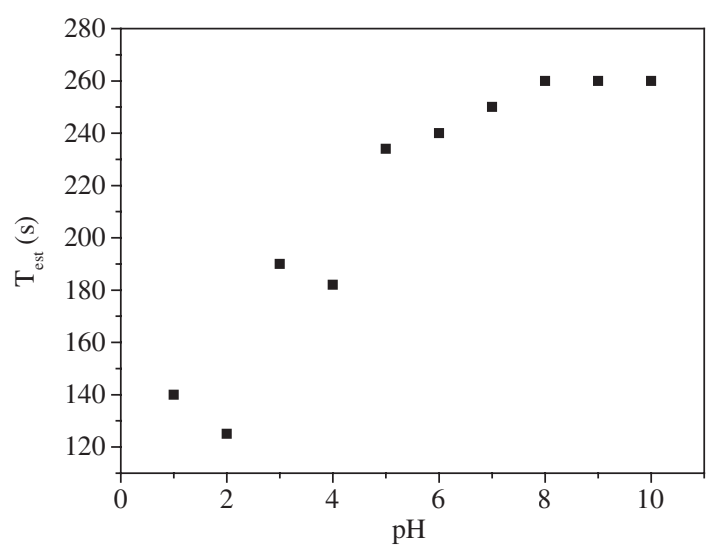

Figura 3. Medidas de tempo de estabilização $\left(\mathrm{T}_{\text {est }}\right)$ do sensor de $\mathrm{pH}$.

desenvolvidos, além de auxiliar nos testes de confiabilidade e de reprodutibilidade dos mesmos.

A Figura 4 mostra o gráfico de corrente de estabilização $\left(\mathrm{I}_{\text {est }}\right)$ vs. pH obtido com o sensor químico. Desse resultado observa-se que $\mathrm{I}_{\text {est }}$ diminui com o $\mathrm{pH}$, partindo de $145 \mathrm{~mA}$ para $\mathrm{pH}=1$ até atingir seu valor de saturação em torno de 1 $\mathrm{mA}$ para $\mathrm{pH}$ maiores que 6 , além de resposta linear de corrente entre $\mathrm{pH}=1$ e 5 . Logo, pode-se concluir que, baseado nos resultados apresentados nas Figura 3 e 4, quanto maior a corrente do dispositivo, isto é, seu nível de dopagem, menor seu tempo de estabilização, como era de se esperar para um sistema que migra de isolante para condutor.

Com o objetivo de avaliar o desempenho do sensor polimérico frente a sua exposição alternada em diferentes soluções químicas, o mesmo foi imerso em quatro soluções aquosas com pH iguais a 1, 3, 5 e 7 em três séries de medidas. Nesse caso o dispositivo era exposto aleatoriamente a uma dessas soluções e, em seguida, exposto as outras três, também aleatoriamente, obedecendo, para tanto, o procedimento experimental apontado em item anterior. A Figura 5 mostra os resultados obtidos. Todavia, é importante destacar que o valor de corrente elétrica do dispositivo é a mesmo para valores de pH entre 7 e 10 e, por isso, não foi apresentado neste trabalho. Os resultados deixam claro que os sensores químicos apresentam boa resposta na faixa de $\mathrm{pH}$ investiga-

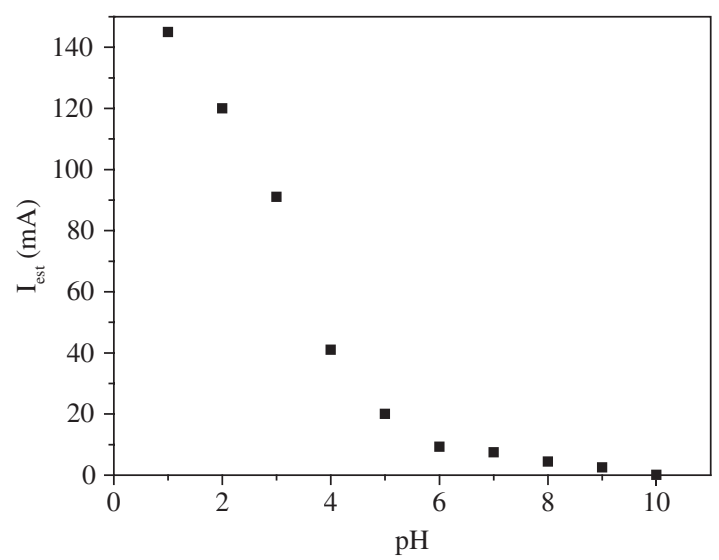

Figura 4. Medidas de corrente $\left(\mathrm{I}_{\mathrm{est}}\right)$ vs. $\mathrm{pH}$ do sensor químico. 


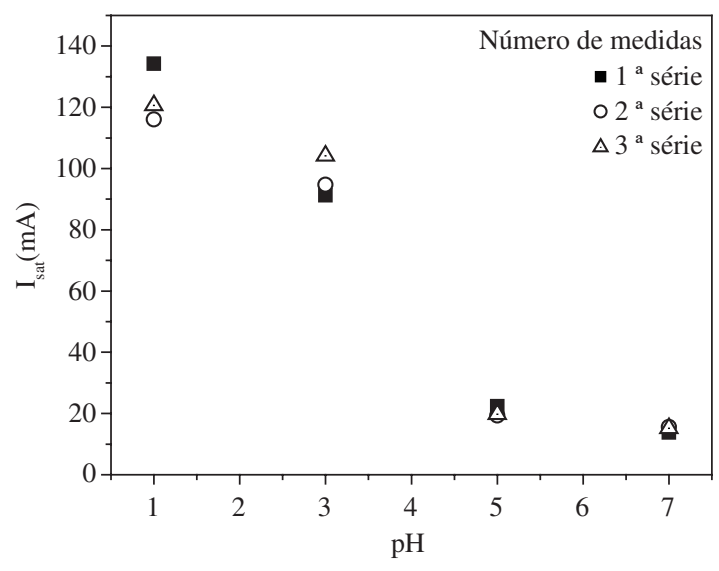

Figura 5. Desempenho do sensor de $\mathrm{pH}$ frente a sua exposição aleatória em diferentes soluções químicas. Três séries alternadas de medidas foram realizadas.

da, com desvio médio inferior a 5\%. Contudo, a resposta do dispositivo é mais precisa para $\mathrm{pH}>4$.

Os gráficos apresentados na Figura 6 mostram a variação percentual da resistência elétrica $(\Delta \mathrm{R}) v s$. ângulo de deflexão $(\theta)$ do sensor polimérico apresentado na Figura 1. Nesse gráfico, $\Delta R=\left(R_{f}-R_{0}\right) / R_{0} \times 100 \%$, onde $R_{f}$ é a resistência elétrica final obtida após a deflexão $\Delta \theta$, enquanto $R_{0}$ é o valor da resistência elétrica inicial para $\theta=0$. É importante destacar que o tempo de estabilização da corrente encontrada para o dispositivo foi de 5 min para todos os ângulos $\Delta \theta$ investigados. Ademais, foram utilizados três filmes de PAni com diferentes níveis de dopagem. O primeiro, totalmente dopado, foi obtido diretamente do processo de polimerização da anilina, enquanto o segundo e o terceiro, moderadamente dopada e desdopada, foram obtidos pós a imersão do primeiro em soluções de $\mathrm{NH}_{4} \mathrm{OH}$ iguais a, respectivamente, 0,1 e $1 \mathrm{M}$.

Os resultados da Figura 6 mostram que a resposta elétrica $\Delta \mathrm{R} v s . \Delta \theta$ do dispositivo apresenta dependência do nível de dopagem da PAni. Para a amostra mais dopada (Figura 6a), $\Delta \mathrm{R}$ para $\Delta \theta=55^{\circ}$ apresenta seu menor valor, $\sim 17 \%$, enquanto, para a amostra desdopada (Figura $6 \mathrm{c}$ ), $\Delta \mathrm{R}$, para a mesma variação de $\Delta \theta$, alcança seu maior valor, $\sim 370 \%$. Por outro lado, quando o dispositivo é investigado variando-se o ângulo $\theta$ de 55 para $0^{\circ}$, a resposta do sistema não é reversível, como mostra a Figura 7. Essa irreversibilidade deve estar relacionado à baixa resistência mecânica à tração observada para compósitos à base de PAni/PET preparados pela técnica in-situ ${ }^{[25]}$. Nesse caso, a deflexão também atua no sentido de romper o filme ultrafino de PAni, aumentando, portando, a resistência elétrica do sistema.

\section{Conclusões}

Neste trabalho foram apresentados os métodos de preparação e a caracterização elétrica de sistemas eletrônicos flexíveis à base de PAni e PET. Os dispositivos foram investigados buscando-se mostrar o potencial de aplicação dos mesmos como dispositivos de monitoramento de $\mathrm{pH}$ e de deflexão

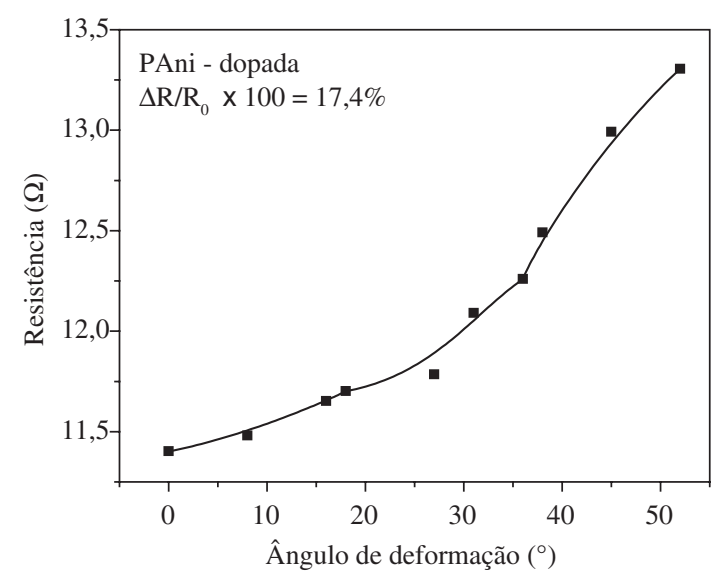

(a)

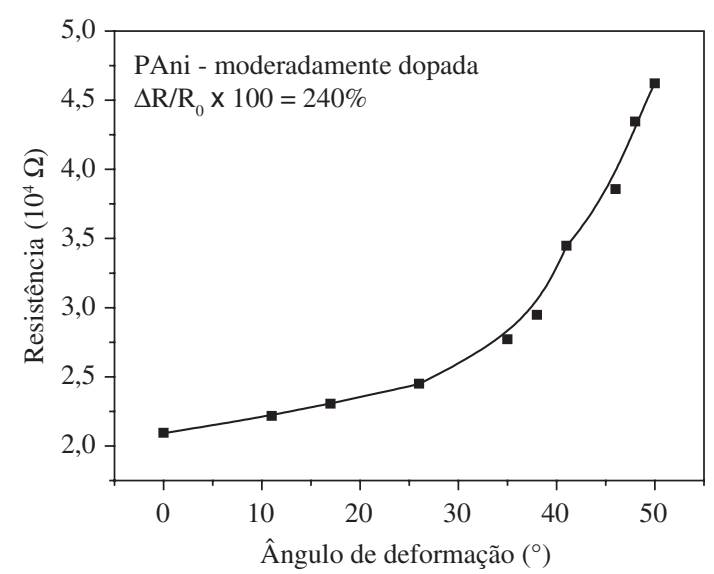

(b)

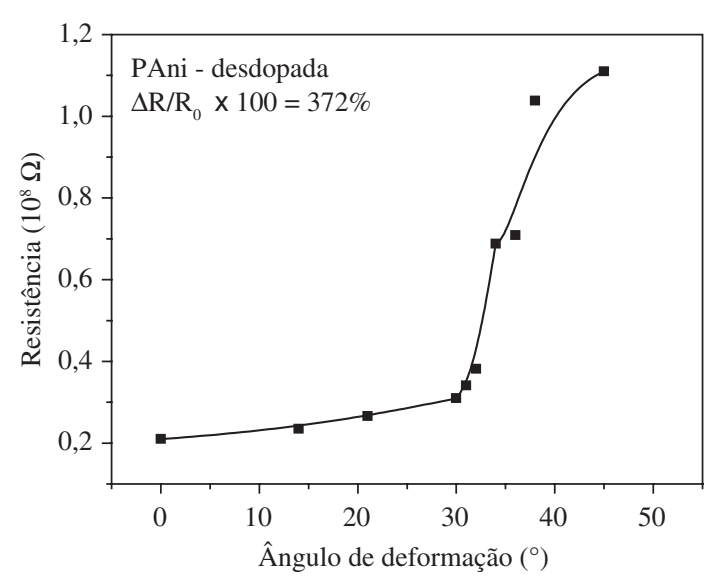

(c)

Figura 6. Variações percentuais da resistência elétrica $(\Delta \mathrm{R})$ vs. ângulo de deflexão $(\Delta \theta)$ do sensor polimérico obtidas a partir de filmes de PAni com diferentes níveis de dopagem.

mecânica. Os resultados obtidos mostraram que o tempo de resposta dos sensores é longo e depende do nível de dopagem da polianilina. De acordo com a aplicação e da faixa de operação dos sensores, eles ainda apresentam características reversíveis ou irreversíveis, linearidade ou não. Levando-se em conta a facilidade e a inovação dos sistemas preparados, o desempenho elétrico dos sensores é de grande interesse da 


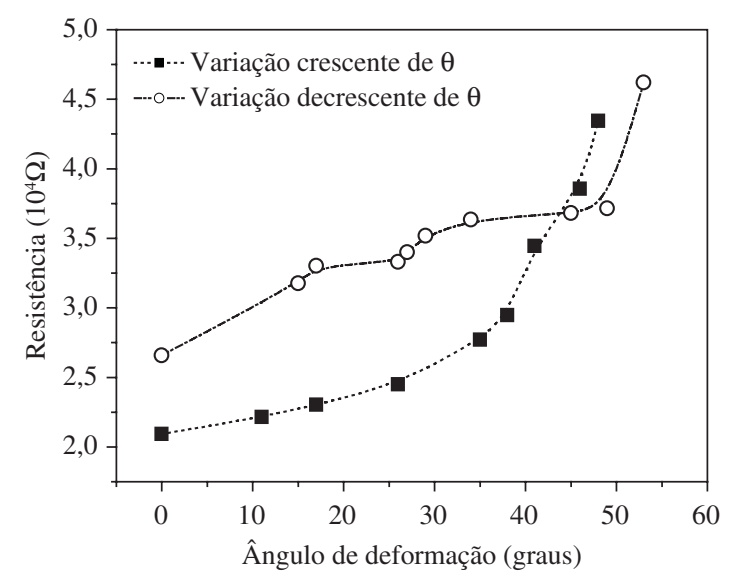

Figura 7. Variações percentuais da resistência elétrica $(\Delta \mathrm{R}) v s$. ângulo de deflexão $(\Delta \theta)$ do sensor polimérico obtidas a partir de variações crescente e decrescentes de $\theta$.

indústria de dispositivos eletrônicos descartáveis, sobretudo pelas simplicidades de processamento e de manufatura.

\section{Agradecimentos}

Os autores agradecem à FAPEMIG, o CNPq e o Instituto Multidisciplinar de Materiais Poliméricos (IMMP/CNPq) pelo auxílio e suporte financeiro que foram essenciais para o desenvolvimento deste trabalho.

\section{Referências Bibliográficas}

1. Chiang, C. K.; Fincher, C. R.; Park, Y. W.; Heeger, A. J.; Shirakawa, H.; Louis, E. J.; Grau, S. C. \& MacDiarmid, A. G. - Phys. Rev. Let., 39, p.1098 (1977).

2. Shirakawa, H. - Synt. Met., 125, p.3 (2002).

3. Nalwa, H. S. - "Organic conductive molecules and polymers", $1^{a}$ ed., John Wiley \& Sons (1997).

4. Skothein, T. A. - "Handbook of conducting polymers", Vol. 1, 1 ${ }^{\mathrm{a}}$ ed., Marcel Dekker Inc. (1986).

5. Akcelrud, L. - Prog. Pol. Sci., 28, p.875 (2003)

6. Pope, M. \& Swenberg, C. E. - "Electronic process in organic crystals and polymers", Clarenfon press, $2^{\text {nd }}$ ed., Oxford (1999).

7. McNeill, C. R.; Abrusci, A.; Zaumseil, J.; Wilson, R.; McKiernan, M. J.; Burroughes, J. H.; Halls, J. J. M.; Greenham, N. C. \& Friend, R.H. - Appl. Phys. Lett., 90, p.193506 (2007).

8. Arbizzani, C.; Mastrogostino, M. \& Scrosati, B. - "Conducting polymers for batteries, supercapacitors and optical devices", in: Organic Conductive Molecules and polymers, cap. 5, p.595, Nalwa H. S. (ed.), John Wiley \& Sons (1997).

9. Adhikari, B. \& Majumdar, S. - Prog. Pol. Sci., 29, p.699 (2004).
10. Myler, S.; Collyer, S. D.; Bridge, K. A. \& Higson, S. P. J. - Biosens Bioelectron, 17, p.35 (2002).

11. Silva, E. A. B.; Borin, J. F.; Nicolucci, P.; Graeff, C. F. O.; Ghilardi Netto, T. \& Bianchi, R. F. - Appl. Phys. Lett., 86, p.131902 (2005).

12. Xie, D.; Jiang, Y.; Pan, W.; Li, D.; Wu, Z. \& Li, Y. - Sens. Act. B, 81, p.158 (2002).

13. Riul Jr., A.; Soto, A. M. G.; Mello, S. V.; Bone, S.; Taylor, D. M. \& Mattoso, L. H. C. - Synth. Met., 132, p.109 (2003).

14. MacDiarmid, A. G.; Chiang, J. C.; Richter, A. F.; Somasiri, N. L D. \& Epstein, A. J. - in: Conducting Polymers, L. Alcacer, (ed.), Reidel, Dordrecht, p.105 (1985).

15. Angelopoulos, M.; Ray, A.; MacDiarmid, A. G. \& Epstein, A. J. - Synth. Met., 21, p.21 (1987).

16. Angelopoulos, M.; Asturias, G. E.; Ermer, S. P.; Ray, A.; Scherr, E. M. \& MacDiarmid, A. G. - Mol. Cryst. Liq. Cryst., 160, p.151 (1988).

17. Travain, S. A.; Libardi, L. H.; Marletta, A.; Giacometti, J. A.; Guimarães, F. E. G. \& Faria, R. M. - Mol. Cryst. Liq. Cryst., 374, p.439 (2002)

18. Bianchi, R. F.; Onmori, R. K.; Goncalves, D.; de Andrade, A. M.; Faria, R. M. \& Irene, E. A. - Synt. Met., 121, 1-3, p.1687 (2001).

19. Bianchi, R. F.; Onmori, R. K. \& Faria, R. M. - J. Pol. Sci. B: Pol. Phys., 43, 1, p.74 (2005).

20. Smits, F. M. - The Bell System Tech. J., 20, p.711 (1958).

21. Sze, S. M. - in: "Physics of semiconductor devices", p.325, $2^{a}$ ed., John Wiley \& Sons (1981).

22. Bianchi, R. F.; Cunha, H. N.; Faria, R. M.; Leal Ferreira, G. F. \& Neto, J. M. G. - J. Phys. D: Appl. Phys., 38, p.1437 (2005).

23. Bianchi, R. F.; Leal Ferreira, G. F.; Lepienski, C. M. \& Faria, R. M. - J. Chem. Phys., 110, p.4602 (1999).

24. Angelopoulous, M.; Asturias, S. P.; Emer, A. R.; Scherr, E. M.; MacDiarmid, A. G.; Akhtar, Z. K. \& Epstein, A. J. - Mol. Cryst. Liq. Cryst., 160, p.151 (1988).

25. Pud, A. A.; Tabellout, M., Kassiba, A.; Korzhenko, A. A.; Rogalsky, S. P.; Shapoval, G. S.; Houzé, F.; Schneegans, O. \& Emery, J. R. - J. Mat. Sci., 36, p.3355 (2001).

Enviado: $27 / 02 / 07$

Reenviado: 20/07/07

Aceito: $10 / 08 / 07$ 